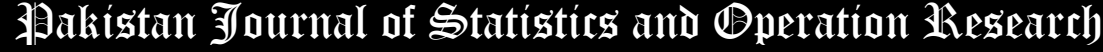

\section{Group Acceptance Sampling Plan for Resubmitted Lots: Size Biased Lomax Distribution}

\author{
Srinivasa Rao Gadde ${ }^{*}$, A. Naga Durgamamba ${ }^{2}$ \\ * Corresponding Author
}

1. Department of Statistics, School of Mathematical and Computer Sciences, Dilla University, Dilla, Ethiopia, gaddesrao@yahoo.com

2. Department of Basic Science and Humanities, Raghu Institute of Technology, Andhra Pradesh, India, durgamamba@gmail.com

\begin{abstract}
This research reveals a group acceptance sampling plan (GASP) for lot resubmitting is designed for conditions wherein an item life is taken from the size biased Lomax distribution (SBLD). The plan parameters of the GASP are obtained by fulfilling the prefixed producer's and consumer's risks as per the test completion time and the number of testers. The projected plan needs a minimal sample size in comparison with the standard GASP. This proposed plan is justified with an example.
\end{abstract}

Key Words: Consumer's risk, group acceptance sampling plan, size biased Lomax distribution, producer's risk, resubmitted method.

\section{Mathematical Subject Classification: 62F10;62F15;62N05}

\section{Introduction}

Acceptance or rejection of an item is dependent on its utility. The top-notch checking in quality control is of various models, one of them is the acceptance sampling scheme. The simple acceptance sampling scheme is well known among the rest of the sampling schemes because of its easy implementation in practical applications. The main hassle in acceptance sampling schemes is to identify the sample size from the available lots. In a simple sampling scheme, the result of acceptance or non-acceptance of a lot depends only on one inspection. These days, items are manufactured to be top-notch quality. While gathering lifetime details of an item, items must pass through a destructive life test. To study the whole lifetime of a higher reliability unit, the life test must be finished within a stipulated span to shorten the experimental time is named as truncated life test. Several authors who developed single sampling schemes under a truncated life test are Baklizi (2003), Balakrishnan et al. (2007), Ravikumar et al. (2016), Rao et al. (2008), Rao and Kantam (2010), Subbarao et al. (2014).

The acceptance sampling scheme that incorporates several units at the same period installed a tester could be named GASP, which decreases the testing time in addition to the cost can be saved with the aid of trying out those items simultaneously. In GASP the sample size ' $n$ ' is designed as $n=r g$ where $r$ is the number of testers and $g$ is the number of groups. If more than $c$ failures occurred in any group at some point in the test time then the test is truncated. GASP enforced on these truncated life tests is termed as GASP under truncated life tests. In these models, obtaining the sample size is equal to obtaining the number of groups. GASP under truncated life tests depending on various models have been discussed in recent time by Aslam et al. (2009), (2011), Aslam and Jun (2009a), (2009b), Rao (2009a),(2009b), Rao et al. (2014).

In several circumstances, the customer cannot accept the given lot depending on a simple acceptance sampling scheme. The manufacturer can conflict with the initial sample data, choosing the next sample of identical size for review and also conclude by eliminating the earlier solutions. Therefore, the discarded lot might be resubmitted, this 
technique is named as resubmitted acceptance sampling scheme. The effectiveness of the re-sampling process with a simple sampling scheme for testing resubmitted lot is not like from the same old acceptance sampling schemes as suggested by Govindaraju and Ganesalingam (1997). Numerous authors who have developed GASP for resubmitted lots are Aslam et al. (2011), Mughal et al. (2015), Rao and Rao (2014), Rosaiah et al. (2017), Rao and Naidu (2016), Rao et al. (2019), Chiang et al. (2018).

\section{Design of GASP for resubmitted lots}

Sampling review for the resubmitted lots is dependent on the following assumptions:

Enduring by the provisions of a written agreement or act, the knowledge of the initial scrutiny leading to rejection is needed to cancel the lot.

The manufacturer is sincere and therefore the client has hopefulness with the producer who won't purposely make the most of re-sampling.

The working process of the projected plan is

Step1:

Carry out the first group sampling assessment by taking $n$ things arbitrarily and allocating them into $g$ groups equally with the condition that every group has to contain $r$ things so that $n=r g$.

Determine the experimentation time, $t_{0}$, and the acceptance range $c$. The lot would be accepted if the failure from all the groups is no more than the acceptance range $c$ at time $t_{0}$; else, it is rejected.

Truncate the test once the failures from each group are more than $c$ or before $t_{0}$, whichever happens, earliest.

Step 2:

Implement the proposed GASP no more than $m$ times on a lot that was refused in the previous step, and turn down the lot on the $m^{\text {th }}$ review if it could not be approved before or at the $(m-1)^{t h}$ re-submission. In this case, the proposed GASP is the original GASP as re-submission is allowed.

The present work suggests a special design for certain conditions and is given below.

When the number of testers $r$ is known, determine the size of group g. Pick $(r \times g)$ goods from a lot and assign $r$ testers to each of the $g$ groups.

In the life test, $(r \times g)$ the sample size is needed.

From every group find the acceptance range $c$ and indicate termination time $t_{0}$.

If the number of failures in any group is larger than $c$ or $(c+1)$ the life test should be shortened and the lot should be declined.

The producer has to honor the consumer's trait and does not use the benefit of the re-sampling. On the refusal of the Primary GASP, execute steps (1) to (4); repeat the projected scheme m times. If the submitted lot fails to pass the $(m-1)^{\text {th }}$ re-submission, it will be refused.

The proposed scheme for the resubmitted lot is not an expansion of the present plan but depends on number of the failures. Govindaraju and Ganesalingam (1997) presented a sampling analysis for resubmitted lots, which cannot be compared to the proposed sampling scheme.Despite the method of resubmitted lots introduced at two-stage and the multiple-stage GASP under truncated life tests, the GASP under a truncated life test can be adopted because of its simplicity of execution. When the lifespan of an item is taken from SBLD in this work, a GASP for resubmitted lots is added.

The use and applications of statistical science's size biased distributions in research related to reliability, biomedicine, ecology, and a variety of other areas are of enormous practical importance in global Science and Technology

The SBLD's probability density function (pdf) and cumulative density function (cdf) are as follows:

$$
\begin{gathered}
f(t)=\frac{\lambda(\lambda-1)}{\theta} \frac{t}{\theta}\left(1+\frac{t}{\theta}\right)^{-(\lambda+1)} \text { for } t \geq 0, \lambda>1, \theta>0 \\
F(t)=1-\left(1+\frac{\lambda t}{\theta}\right)\left(1+\frac{t}{\theta}\right)^{-\lambda} ; t \geq 0, \lambda>1, \theta>0
\end{gathered}
$$

where $\lambda$ is shape and $\theta$ is scale parameters of the model. The mean and variance of SBLD is

$$
\text { Mean }=\mu=\frac{2 \theta}{\lambda-2} \text { for } \lambda>2
$$




$$
\text { Variance }=\sigma^{2}=\frac{2 \theta^{2} \lambda}{(\lambda-2)^{2}(\lambda-3)} \text { for } \lambda>3
$$

We're using the known experiment time $t_{0}$ and number of testers' $r$ to derive the unknown GASP parameters for resubmitted lots, where the producer's and consumer's risks are predefined.

Assume that the parameter $\lambda$ is pre-defined and that the mean value shown in Equation (3) is solely dependent on the scale parameter $\theta$.The probability of accepting a lot for the standard GASP is mentioned as

$$
L(P)=\sum_{i=0}^{c}\left(\begin{array}{c}
r g \\
i
\end{array}\right) p^{i}(1-p)^{r g-i}
$$

where $r$ denotes the number of testers, $c$ denotes the acceptance range, $g$ denotes the group size and $p$ denotes the probability that an item will fail in either group during the test completion period $t_{0}$. The test termination time $t_{0}$ is given in Equation (6)

$$
t_{0}=a \mu_{0}
$$

where $\mu_{0}$ is specified average life and a is test termination ratio. Then the lot acceptance probability for SBLD is in (7)

$$
p=F\left(t_{0}\right)=1-\left(\frac{2 \lambda a}{\left(\mu / \mu_{0}\right)(\lambda-2)}+1\right)\left[1+\frac{2 a}{\left(\mu / \mu_{0}\right)(\lambda-2)}\right]^{-\lambda}
$$

where $\mu / \mu_{0}$ is a ratio of true mean life and the specified mean life.

According to Govindaraju and Ganesalingam (1997), the chance of a lot of acceptance for resubmitted lots with $m$ - 1 resubmissions is

$$
P_{a}(a)=1-(1-L(P))^{m}
$$

In equation (9) the OC function is discussed

$$
P_{a}(a)=1-\left(1-\sum_{i=0}^{c}\left(\begin{array}{c}
r g \\
i
\end{array}\right) p^{i}(1-p)^{r g-i}\right)^{m}
$$

We must calculate the unknown values $c$ and $g$ for the projected GASP of resubmitted lots with a known number of testers $r$ and a predefined truncated life test time $t_{0}=a \mu_{0}$, where $a$ is the termination time and $\mu_{0}$ is the mean of the SBLD. The lot is considered to be fine if the true average lifetime $\mu$ is $\geq t_{0}$ the target one, else it is bad. There are two risks in acceptance sampling schemes: one for the producer and one for the consumer. Producer's risk, $\alpha$, is rejecting a lot when it is good and consumer's risk, $\beta$, is accepting a lot when it is bad. Both producers and consumers require a sampling scheme to take a decision that will strike a balance between their concern risks. At the acceptance probability level (ARL), the minimum lot acceptance probability 1- $\alpha$ is required for producers, and the maximum lot acceptance probability $\beta$ is required for consumers at the lot tolerance reliability level (LTRL).

In equation (7) if we substitute, the values $\mu / \mu_{0}=2(2) 10$ will get the probability of failure $P_{1}$ of the producer's risk at ARL and the value $\mu / \mu_{0}=1$ will get the probability of failure $P_{2}$ of the consumer's risk at LTRL respectively.

Solve the inequalities given in (10) and (11) at the same time so that we get the values $c$ and $g$ for the proposed plan.

$$
1-\left(1-\sum_{i=0}^{c}\left(\begin{array}{c}
r g \\
i
\end{array}\right) p_{1}^{i}\left(1-p_{1}\right)^{r g-i}\right)^{m} \geq 1-\alpha
$$

and

$$
1-\left(1-\sum_{i=0}^{c}\left(\begin{array}{c}
r g \\
i
\end{array}\right) p_{2}^{i}\left(1-p_{2}\right)^{r g-i}\right)^{m} \leq \beta
$$

where 


$$
p_{1}=1-\left(\frac{2 \lambda a}{\left(\mu / \mu_{0}\right)(\lambda-2)}+1\right)\left[1+\frac{2 a}{\left(\mu / \mu_{0}\right)(\lambda-2)}\right]^{-\lambda}
$$

and

$$
p_{2}=1-\left(\frac{2 \lambda a}{(\lambda-2)}+1\right)\left[1+\frac{2 a}{(\lambda-2)}\right]^{-\lambda}
$$

To estimate the two parameters $g$ and $c$ of the proposed GASP consider the producer's risk $\alpha=0.05$ and termination time $t_{0}=a \mu_{0}$ where $a=0.3,0.5$, with the predefined time $t_{0}$, at resubmitted lot $m=2,3$ are determined as per the consumer's confidence levels $\beta=0.25,0.10,0.05$ or 0.01 . The SBLD parameter $\lambda=3$ and 4 at $m=2$, the values of the parameters are presented in Tables 1 and 2. Also for $m=2$ and the estimated parameter value $\lambda=2.96$ for the true mean lifetime, the parameter values are mentioned in Table 3. The SBLD parameter $\lambda=3$ and 4 at $m=3$, the parameters are presented in Tables 4 and 5. Also for $m=3$ and the estimated parameter value $\lambda=2.96$ for the true mean lifetime, the parameter values are mentioned in Table 6 . Tables 1,2, 4, and 5 for the proposed GASP show that as ' $a$ ' rises from 0.3 to 0.5 at given $\beta$, the required number of groups $(g)$ decreases and the acceptance range (c) increases; $\mu / \mu_{0}$ and $r$ remain constant.

Tables 3 and 6 show, however, that the number of groups $(g)$ is reducing and the acceptance range, $c$, is improving as $a$ rises from 0.3 to 0.5 . The FORTRAN coding is also provided in appendix.

$$
<<<\text { Tables 1-6 are around here }>>
$$

\section{Projected Scheme is Applied to Industry}

Using real data from Khalil (2019), the active repair times (hours) for an airborne communication transceiver (source: Chhikara and Folks (1989)) are presented. This data set has been resubmitted and is available as follows. $0.2,0.3,0.5,0.5,0.5,0.5,0.6,0.6,0.7,0.7,0.7,0.8,0.8,1.0,1.0,1.0,1.0,1.1,1.3,1.5,1.5,1.5,1.5,2.0,2.0,2.2$, 2.5, 2.7, 3.0, 3.0, 3.3, 3.3, 4.0, 4.0, 4.5, 4.7, 5.0, 5.4, 5.4, 7.0, 7.5, 8.8, 9.0, 10.3, 22.0, 24.5

Figure 1 depicts the goodness of fit of the data using empirical density and the p-p plot for SBLD. Using the Kolmogorov-Smirnov test, we found that the maximum distance between the data and the fitted SBLD for active repair times (hours) for an airborne communication transceiver is 0.11129 with a p-value of 0.6191 . As a result, the SBLD is a good fit for the active repair times (hours) of an airborne communication transceiver.

Since the specified lifetime of an item is derived from SBLD using the estimated parameter, $\lambda=2.306$. Assume that it is necessary to form the GASP to ensure that the true average lifetime is greater than the active repair times (hours) for an airborne communication transceiver 0.2 through the experiment to be completed the active repair times (hours) for an airborne communication transceiver by 0.80 to protect the producer's risk at 0.05 . Since $\lambda=$ 2.306, the consumer's risk $\beta=0.25, r=5, a=0.3$, and $\mu / \mu_{0}=4$, the minimum number of groups $g=10$ and acceptance range $c=9$ are calculated from Table 3. As a result, the sampling scheme can be implemented as follows: Choose 50 items in total, then assign five testers to each of the ten groups. If there are more than nine failures below active repair times (hours) for an airborne communication transceiver 0.80, we reject the lot. Because there are more than nine failures before the termination time, active repair times (hours) 0.80 , the active repair times (hours) for an airborne communication transceiver could have been rejected.

\section{Conclusions}

According to the study, a GASP for a resubmitted lot with a specific average life has been discussed for the SBLD. The projected sampling scheme estimates $c$ and $g$ are obtained in such a way that the lot acceptance probability is higher than 1- $\alpha$ at the producer indicated standard but less than $\beta$ at the customer defined standard. Based on a variety of estimates and mean values, the tables are designed for industrialized applications. It was discovered that the required number of groups rises as customer confidence grows or the number of re-submissions reduces. More specifically, by allocating a few re-submissions in the projected sampling scheme, the sample range can be significantly reduced. 

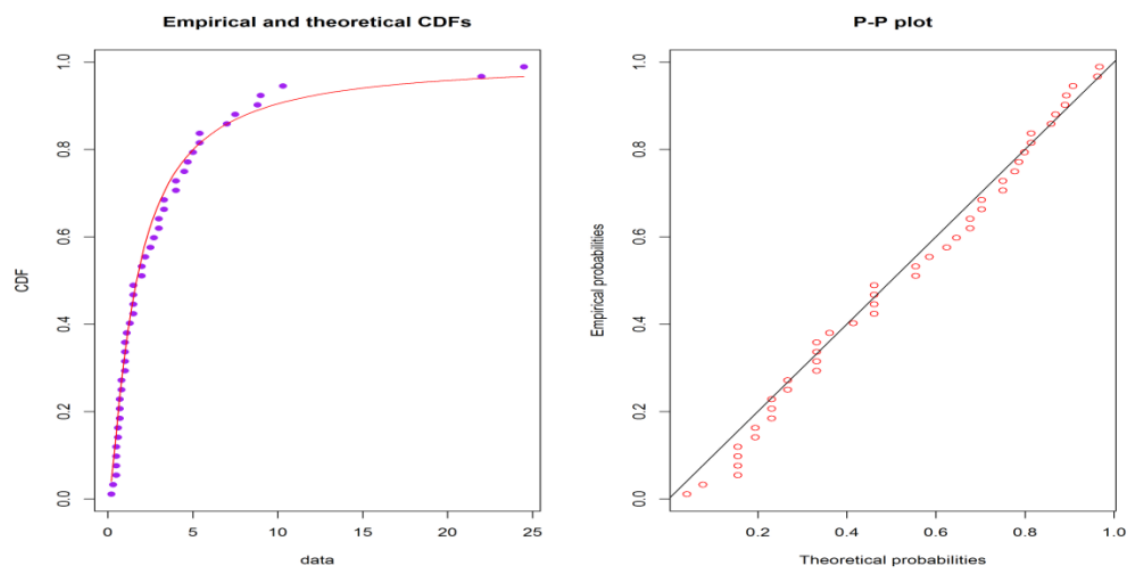

Figure 1: Empirical and theoretical CDFs for active repair times (hours) for an airborne communication transceiver, as well as a P-P plot.

\section{References}

1. Aslam, M., Jun, C.H., and Ahmad, M. (2009). A group sampling plan based on truncated life tests for gamma distribution, Pakistan Journal of Statistics, 25, 333-340.

2. Aslam, M., and Jun, C.H. (2009a). A group sampling plan based on truncated life test having Weibull distribution, Journal of Applied Statistics,36, 1021-1027.

3. Aslam, M., and Jun, C.H. (2009b) A group acceptance sampling plans for truncated life tests based on the Inverse Rayleigh and Log-Logistic distributions, Pakistan Journal of Statistics, 25(2), 107-119.

4. Aslam, M., Jun, C.H., Lio, Y.L., Ahmad, M., and Rasool, M. (2011). Group acceptance sampling plans for resubmitted lots under Burr-type XII distributions, Journal of Chinese Institute of Industrial Engineers, 28(8), 606-615.

5. Aslam, M., Kundu, D., Jun, C., and Ahmad, M. (2011). Time Truncated Group Acceptance Sampling Plans for Generalized Exponential Distribution, Journal of Testing and Evaluation, 39(4),671-677.

6. Baklizi, A. (2003). Acceptance sampling based on truncated life tests in the Pareto distribution of the second kind, Advances and Applications in Statistics, 3, 33-48.

7. Balakrishnan, N., Leiva, V., and Lopez, J. (2007). Acceptance sampling plans from truncated life tests based on generalized Birnbaum-Saunders distribution, Communications in Statistics-Simulation and Computation,36, 643-656.

8. Chhikara, R. S., and Folks, J. L. (1989). The Inverse Gaussian Distribution. Marcel Dekker, New York.

9. Govindaraju, K., and Ganesalingam, S. (1997). Sampling inspection for resubmitted lots, Commun. Stat. Simulat, 26, 163-1176.

10. Chiang, J-Y, Zhao, X., Yang, L., Wang, X., Tong, W. and Zhao, H. (2018). Group Acceptance Sampling Plans for Resubmitted Lots under the Kumaraswamy Burr XII Distribution, International Journal of Intelligent Technologies \& Applied Statistics, 11(1), 57-72.

11. Khalil, M. G. (2019), A New Distribution For Modeling Extreme Values, Journal of Data Science, 17(3),

12. 81-503.

13. Mughal, A.R., Zain, Z., and Aziz, N. (2015). Group Acceptance Sampling Plan for Re-Submitted Lots Under Generalized Pareto Distribution, Research Journal of Applied Sciences, Engineering and Technology, 10(5), 599-606.

14. Rao, B.S., Kumar, CH.S., and Rosaiah, K. (2014). Group Acceptance Sampling Plans for Life Tests Based on Half Normal Distribution, Sri Lankan Journal of Applied Statistics, 15(3),221-231.

15. Rao, G.S. (2009a). A group acceptance sampling plans for truncated life tests for Marshall-Olkin extended Lomax distribution, Electronic Journal of Applied Statistical Analysis, 3(1), 18-28.

16. Rao, G.S. (2009b). A Group Acceptance Sampling Plans for Lifetimes Following a Generalized Exponential Distribution, Economic Quality Control , 24(1), 75-85.

17. Rao, G.S., Ghitany, M.E., and Kantam, R.R.L. (2008). Acceptance sampling plans for Marshall-Olkin extended Lomax distribution, International Journal of Applied Mathematics, 21, 315- 325. 
18. Rao, G.S., and Kantam, R.R.L. (2010). Acceptance sampling plans from truncated life tests based on loglogistic distribution for percentiles, Economic Quality Control, 25(2), 153-167.

19. Rao, G.S., Rosaiah, K., and Babu, M.S. (2019). Group acceptance sampling plans for resubmitted lots under exponentiated Frechet distribution, International Journal of Computing Science and Mathematics, 10(1), 1121.

20. Rao, G.S., and Naidu, Ch. R. (2016).Group acceptance sampling plans for resubmitted lots under exponentiated half logistic distribution, Journal of Industrial and production Engineering, 33(2), 114-122.

21. Rao, G.S., and Rao, B.S. (2014). Group acceptance sampling plans for resubmitted lots for life tests based on half logistic distribution, Journal of Data Science, 12, 647-660.

22. Ravikumar, M.S., Kantam, R.R.L., and Nagadurgamamba, A. (2016). Acceptance Sampling Plans for Burr Type X Distribution, International Journal of Advanced Technology in Engineering and Science, 4(8), 36-44.

23. Rosaiah, K., Rao, G.S., Kalyani, K. and Sivakumar, D.C.U. (2017), Group acceptance sampling plan for resubmitted lots based on life tests for odds exponential log logistic distribution, International Journal of Quality \& Reliability Management, 34(8), 1343-1351.

24. Subbarao, R., Nagadurgamamba, A. and Kantam, R.R.L. (2014), Acceptance Sampling Plans: Size Biased Lomax Model, Universal Journal of Applied Mathematics, 2(4), 76-183.

\section{Appendix A}

Table 1: GASP for resubmitted lots having $m=2$ for SBLD for $\lambda=3$.

\begin{tabular}{|c|c|c|c|c|c|c|c|c|c|c|c|c|c|}
\hline \multirow{3}{*}{$\beta$} & \multirow{3}{*}{$\mu / \mu_{0}$} & \multicolumn{6}{|c|}{$\mathrm{r}=5$} & \multicolumn{6}{|c|}{$\mathrm{r}=10$} \\
\hline & & \multicolumn{3}{|c|}{$\mathrm{a}=0.3$} & \multicolumn{3}{|c|}{$\mathrm{a}=0.5$} & \multicolumn{3}{|c|}{$\mathrm{a}=0.3$} & \multicolumn{3}{|c|}{$\mathrm{a}=0.5$} \\
\hline & & $c$ & $g$ & $\mathrm{P}_{\mathrm{a}}\left(P_{1}\right)$ & $c$ & $g$ & $\mathrm{P}_{\mathrm{a}}\left(P_{1}\right)$ & $c$ & $g$ & $\mathrm{P}_{\mathrm{a}}\left(P_{1}\right)$ & $c$ & $g$ & $\mathrm{P}_{\mathrm{a}}\left(P_{1}\right)$ \\
\hline \multirow{5}{*}{0.25} & 2 & 5 & 6 & 0.9550 & - & - & - & - & - & - & 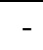 & - & - \\
\hline & 4 & 1 & 2 & 0.9942 & 2 & 3 & 0.9601 & 2 & 3 & 0.9736 & - & - & - \\
\hline & 6 & 0 & 2 & 0.9559 & 1 & 2 & 0.9894 & 0 & 1 & 0.9559 & 3 & 4 & 0.9679 \\
\hline & 8 & 0 & 2 & 0.9829 & 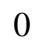 & 1 & 0.9744 & 0 & 1 & 0.9829 & 1 & 2 & 0.9778 \\
\hline & 10 & 0 & 2 & 0.9921 & 0 & 1 & 0.9876 & 0 & 1 & 0.9921 & 0 & 1 & 0.9559 \\
\hline \multirow{5}{*}{0.10} & 2 & 6 & 7 & 0.9653 & - & - & - & & - & - & - & - & - \\
\hline & 4 & 1 & 3 & 0.9766 & 2 & 3 & 0.9601 & 2 & 3 & 0.9736 & - & - & - \\
\hline & 6 & 0 & 2 & 0.9559 & 1 & 2 & 0.9894 & 0 & 1 & 0.9559 & 3 & 4 & 0.9679 \\
\hline & 8 & 0 & 2 & 0.9829 & 0 & 1 & 0.9744 & 0 & 1 & 0.9829 & 1 & 2 & 0.9778 \\
\hline & 10 & 0 & 2 & 0.9921 & 0 & 1 & 0.9876 & 0 & 1 & 0.9921 & 0 & 1 & 0.9559 \\
\hline \multirow{5}{*}{0.05} & 2 & 9 & 10 & 983 & - & - & - & & - & - & - & - & - \\
\hline & 4 & 2 & 4 & 0.9959 & 2 & 3 & 0.9601 & 2 & J & 0.9736 & - & - & _- \\
\hline & 6 & 0 & 2 & 0.9559 & 1 & 2 & 0.9894 & 0 & 1 & 0.9559 & 3 & 4 & 0.9679 \\
\hline & 8 & 0 & 2 & 0.9829 & 1 & 2 & 0.9981 & 0 & 1 & 0.9829 & 1 & 2 & 0.9778 \\
\hline & 10 & 0 & 2 & 0.9921 & 0 & 2 & 0.9559 & 0 & 1 & 0.9921 & 0 & 1 & 0.9559 \\
\hline \multirow{5}{*}{0.01} & 2 & 12 & 14 & 0.9791 & - & - & - & - & - & - & - & - & - \\
\hline & 4 & 2 & 6 & 0.9736 & 2 & 3 & 0.9601 & 2 & 3 & 0.9736 & - & - & - \\
\hline & 6 & 1 & 5 & 0.9869 & 1 & 3 & 0.9598 & 1 & 3 & 0.9763 & 3 & 4 & 0.9679 \\
\hline & 8 & 0 & 3 & 0.9640 & 1 & 3 & 0.9916 & 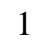 & 3 & 0.9957 & 1 & 2 & 0.9778 \\
\hline & 10 & 0 & 3 & 0.9831 & 0 & 2 & 0.9559 & 0 & 2 & 0.9712 & 0 & 1 & 0.9559 \\
\hline
\end{tabular}


Table 2: GASP for resubmitted lots having $m=3$ for $\operatorname{SBLD}$ for $\lambda=3$.

\begin{tabular}{|c|c|c|c|c|c|c|c|c|c|c|c|c|c|}
\hline \multirow{3}{*}{$\beta$} & \multirow{3}{*}{$\mu / \mu_{0}$} & \multicolumn{6}{|c|}{$r=5$} & \multicolumn{6}{|c|}{$r=10$} \\
\hline & & \multicolumn{3}{|c|}{$\mathrm{a}=0.3$} & \multicolumn{3}{|c|}{$\mathrm{a}=0.5$} & \multicolumn{3}{|c|}{$\mathrm{a}=0.3$} & \multicolumn{3}{|c|}{$\mathrm{a}=0.5$} \\
\hline & & $c$ & $g$ & $\mathrm{P}_{\mathrm{a}}\left(P_{1}\right)$ & $c$ & $g$ & $\mathrm{P}_{\mathrm{a}}\left(P_{1}\right)$ & $c$ & $g$ & $\mathrm{P}_{\mathrm{a}}\left(P_{1}\right)$ & $c$ & $g$ & $\mathrm{P}_{\mathrm{a}}\left(P_{1}\right)$ \\
\hline \multirow{5}{*}{0.25} & 2 & 3 & 4 & 0.9777 & - & - & - & - & - & - & - & - & - \\
\hline & 4 & 1 & 3 & 0.9964 & 1 & 2 & 0.9782 & 1 & 2 & 0.9864 & - & - & - \\
\hline & 6 & 0 & 2 & 0.9907 & 0 & 1 & 0.9848 & 0 & 1 & 0.9907 & 1 & 2 & 0.9717 \\
\hline & 8 & 0 & 2 & 0.9978 & 0 & 1 & 0.9959 & 0 & 1 & 0.9978 & 0 & 1 & 0.9744 \\
\hline & 10 & 0 & 2 & 0.9993 & 0 & 1 & 0.9986 & 0 & 1 & 0.9993 & 0 & 1 & 0.9907 \\
\hline \multirow{5}{*}{0.10} & 2 & 5 & 7 & 0.9630 & - & - & - & - & - & - & - & - & - \\
\hline & 4 & 1 & 3 & 0.9964 & 1 & 2 & 0.9782 & 1 & 2 & 0.9864 & - & - & - \\
\hline & 6 & 0 & 2 & 0.9907 & 0 & 1 & 0.9848 & 0 & 1 & 0.9907 & 1 & 2 & 0.9717 \\
\hline & 8 & 0 & 2 & 0.9978 & 0 & 1 & 0.9959 & 0 & 1 & 0.9978 & 0 & 1 & 0.9744 \\
\hline & 10 & 0 & 2 & 0.9993 & 0 & 1 & 0.9986 & 0 & 1 & 0.9993 & 0 & 1 & 0.9907 \\
\hline \multirow{5}{*}{0.05} & 2 & 5 & 7 & 0.963 & - & - & - & - & - & - & - & - & - \\
\hline & 4 & 1 & 4 & 0.9864 & 1 & 2 & 0.9782 & 1 & 2 & 0.9864 & - & - & - \\
\hline & 6 & 0 & 3 & 0.9736 & 1 & 2 & 0.9989 & 1 & 2 & 0.9995 & 1 & 2 & 0.9717 \\
\hline & 8 & 0 & 3 & 0.9932 & 0 & 2 & 0.9744 & 0 & 2 & 0.9854 & 0 & 1 & 0.9744 \\
\hline & 10 & 0 & 3 & 0.9978 & 0 & 2 & 0.9907 & 0 & 2 & 0.9951 & 0 & 1 & 0.9907 \\
\hline \multirow{5}{*}{0.01} & 2 & 8 & 11 & 0.9663 & - & - & - & - & - & - & - & - & - \\
\hline & 4 & 1 & 5 & 0.9653 & 2 & 4 & 0.9586 & 2 & 3 & 0.9957 & - & - & - \\
\hline & 6 & 0 & 3 & 0.9736 & 1 & 3 & 0.9919 & 1 & 3 & 0.9963 & 1 & 2 & 0.9717 \\
\hline & 8 & 0 & 3 & 0.9932 & 0 & 2 & 0.9744 & 0 & 2 & 0.9854 & 0 & 1 & 0.9744 \\
\hline & 10 & 0 & 3 & 0.9978 & 0 & 2 & 0.9907 & 0 & 2 & 0.9951 & 0 & 1 & 0.9907 \\
\hline
\end{tabular}

Table 3: GASP for resubmitted lots having $m=3$ for SBLD for $\lambda=3$.

\begin{tabular}{|c|c|c|c|c|c|c|c|}
\hline \multirow[t]{2}{*}{$\beta$} & \multirow[t]{2}{*}{$\mu / \mu_{0}$} & \multicolumn{3}{|c|}{$\begin{array}{c}r=5 \\
a=0.3\end{array}$} & \multicolumn{3}{|c|}{$\begin{array}{c}\mathrm{r}=10 \\
\mathrm{a}=0.3\end{array}$} \\
\hline & & $c$ & $g$ & $\mathrm{P}_{\mathrm{a}}\left(P_{1}\right)$ & $c$ & $g$ & $\mathrm{P}_{\mathrm{a}}\left(P_{1}\right)$ \\
\hline \multirow{5}{*}{0.25} & 2 & - & - & - & - & - & - \\
\hline & 4 & 9 & 10 & 0.9541 & - & - & - \\
\hline & 6 & 1 & 2 & 0.9551 & - & - & - \\
\hline & 8 & 1 & 2 & 0.9891 & 3 & 4 & 0.9665 \\
\hline & 10 & 0 & 1 & 0.9674 & 1 & 2 & 0.9666 \\
\hline \multirow{5}{*}{0.10} & 2 & - & - & - & - & - & - \\
\hline & 4 & 9 & 10 & 0.9541 & - & - & - \\
\hline & 6 & 1 & 2 & 0.9551 & - & - & - \\
\hline & 8 & 1 & 2 & 0.9891 & 3 & 4 & 0.9665 \\
\hline & 10 & 0 & 1 & 0.9674 & 1 & 2 & 0.9666 \\
\hline \multirow{5}{*}{0.05} & 2 & - & - & - & - & - & - \\
\hline & 4 & 9 & 10 & 0.9541 & - & - & - \\
\hline & 6 & 1 & 2 & 0.9551 & - & - & - \\
\hline & 8 & 1 & 2 & 0.9891 & 3 & 4 & 0.9665 \\
\hline & 10 & 0 & 1 & 0.9674 & 1 & 2 & 0.9666 \\
\hline \multirow{3}{*}{0.01} & 2 & - & - & - & - & - & - \\
\hline & 4 & 9 & 10 & 0.9541 & - & - & - \\
\hline & 6 & 1 & 2 & 0.9551 & - & - & - \\
\hline
\end{tabular}




\begin{tabular}{ccccccc}
8 & 1 & 2 & 0.9891 & 3 & 4 & 0.9665 \\
10 & 1 & 2 & 0.9969 & 1 & 2 & 0.9666 \\
\hline
\end{tabular}

Table 4: GASP for resubmitted lots having $m=2$ for SBLD for $\lambda=4$.

\begin{tabular}{|c|c|c|c|c|c|c|c|c|c|c|c|c|c|}
\hline \multirow{3}{*}{$\beta$} & \multirow{3}{*}{$\mu / \mu_{0}$} & \multicolumn{6}{|c|}{$r=5$} & \multicolumn{6}{|c|}{$r=10$} \\
\hline & & \multicolumn{3}{|c|}{$\mathrm{a}=0.3$} & \multicolumn{3}{|c|}{$\mathrm{a}=0.5$} & \multicolumn{3}{|c|}{$\mathrm{a}=0.3$} & \multicolumn{3}{|c|}{$\mathrm{a}=0.5$} \\
\hline & & $c$ & $g$ & $\mathrm{P}_{\mathrm{a}}\left(P_{1}\right)$ & $c$ & $g$ & $\mathrm{P}_{\mathrm{a}}\left(P_{1}\right)$ & $c$ & $g$ & $\mathrm{P}_{\mathrm{a}}\left(P_{1}\right)$ & $c$ & $g$ & $\mathrm{P}_{\mathrm{a}}\left(P_{1}\right)$ \\
\hline \multirow{5}{*}{0.25} & 2 & 4 & 7 & 0.9698 & - & - & - & - & - & - & - & - & - \\
\hline & 4 & 1 & 3 & 0.9965 & 1 & 2 & 0.9832 & 1 & 2 & 0.9904 & 4 & 5 & 0.9557 \\
\hline & 6 & 0 & 2 & 0.9855 & 0 & 1 & 0.9775 & 0 & 1 & 0.9855 & 1 & 2 & 0.9823 \\
\hline & 8 & 0 & 2 & 0.9948 & 0 & 1 & 0.9915 & 0 & 1 & 0.9948 & 0 & 1 & 0.9690 \\
\hline & 10 & 0 & 2 & 0.9977 & 0 & 1 & 0.9961 & 0 & 1 & 0.9977 & 0 & 1 & 0.9855 \\
\hline \multirow{5}{*}{0.10} & 2 & 5 & 9 & 0.9671 & - & - & - & - & - & - & - & - & - \\
\hline & 4 & 1 & 4 & 0.9904 & 1 & 2 & 0.9832 & 1 & 2 & 0.9904 & 4 & 5 & 0.9557 \\
\hline & 6 & 0 & 3 & 0.9693 & 1 & 2 & 0.9985 & 1 & 2 & 0.9993 & 1 & 2 & 0.9823 \\
\hline & 8 & 0 & 3 & 0.9887 & 0 & 2 & 0.9690 & 0 & 2 & 0.9806 & 0 & 1 & 0.9690 \\
\hline & 10 & 0 & 3 & 0.995 & 0 & 2 & 0.9855 & 0 & 2 & 0.9913 & 0 & 1 & 0.9855 \\
\hline \multirow{5}{*}{0.05} & 2 & 6 & 11 & 0.9657 & - & - & - & - & - & - & - & - & - \\
\hline & 4 & 1 & 5 & 0.9799 & 2 & 3 & 0.9957 & 1 & 3 & 0.9643 & 4 & 5 & 0.9557 \\
\hline & 6 & 0 & 3 & 0.9693 & 1 & 3 & 0.9934 & 1 & 3 & 0.9969 & 1 & 2 & 0.9823 \\
\hline & 8 & 0 & 3 & 0.9887 & 0 & 2 & 0.9690 & 0 & 2 & 0.9806 & 0 & 1 & 0.9690 \\
\hline & 10 & 0 & 3 & 0.995 & 0 & 2 & 0.9855 & 0 & 2 & 0.9913 & 0 & 1 & 0.9855 \\
\hline \multirow{5}{*}{0.01} & 2 & 9 & 17 & 0.9653 & - & - & - & - & - & - & - & - & - \\
\hline & 4 & 1 & 6 & 0.9643 & 2 & 4 & 0.9829 & 1 & 3 & 0.9643 & 4 & 5 & 0.9557 \\
\hline & 6 & 1 & 6 & 0.9969 & 1 & 3 & 0.9934 & 1 & 3 & 0.9969 & 1 & 2 & 0.9823 \\
\hline & 8 & 0 & 5 & 0.9708 & 1 & 3 & 0.9989 & 0 & 3 & 0.9595 & 1 & 2 & 0.9969 \\
\hline & 10 & 0 & 5 & 0.9867 & 0 & 3 & 0.9693 & 0 & 3 & 0.9813 & 1 & 2 & 0.9993 \\
\hline
\end{tabular}

Table 5: GASP for resubmitted lots having $m=3$ for SBLD for $\lambda=4$

\begin{tabular}{cccccccccccccc}
\hline \multicolumn{1}{c}{$\beta$} & $\mu / \mu_{0}$ & \multicolumn{1}{c}{$\mathrm{a}=0.3$} & \multicolumn{1}{c}{$\mathrm{a}=0.5$} & \multicolumn{1}{c}{$\mathrm{a}=0.3$} & & $\mathrm{a}=0.5$ \\
& & $c$ & $g$ & $\mathrm{P}_{\mathrm{a}}\left(P_{1}\right)$ & $c$ & $g$ & $\mathrm{P}_{\mathrm{a}}\left(P_{1}\right)$ & $c$ & $g$ & $\mathrm{P}_{\mathrm{a}}\left(P_{1}\right)$ & $c$ & $g$ & $\mathrm{P}_{\mathrm{a}}\left(P_{1}\right)$ \\
\hline \multirow{4}{*}{0.25} & 2 & 2 & 5 & 0.9534 & 16 & 17 & 0.9515 & 8 & 9 & 0.9537 & - & - & - \\
& 4 & 0 & 2 & 0.9869 & 0 & 1 & 0.9781 & 0 & 1 & 0.9869 & 1 & 2 & 0.9510 \\
& 6 & 0 & 2 & 0.9983 & 0 & 1 & 0.9966 & 0 & 1 & 0.9983 & 0 & 1 & 0.9787 \\
& 8 & 0 & 2 & 0.9996 & 0 & 1 & 0.9992 & 0 & 1 & 0.9996 & 0 & 1 & 0.9946 \\
& 10 & 0 & 2 & 0.9999 & 0 & 1 & 0.9998 & 0 & 1 & 0.9999 & 0 & 1 & 0.9983 \\
& 2 & 3 & 7 & 0.9577 & 16 & 17 & 0.9515 & 8 & 9 & 0.9537 & - & - & - \\
& 4 & 0 & 3 & 0.9633 & 1 & 3 & 0.9850 & 1 & 3 & 0.9932 & 1 & 2 & 0.9510 \\
0.10 & 6 & 0 & 3 & 0.9946 & 0 & 2 & 0.9787 & 0 & 2 & 0.9884 & 0 & 1 & 0.9787 \\
& 8 & 0 & 3 & 0.9988 & 0 & 2 & 0.9946 & 0 & 2 & 0.9973 & 0 & 1 & 0.9946 \\
& 10 & 0 & 3 & 0.9996 & 0 & 2 & 0.9983 & 0 & 2 & 0.9992 & 0 & 1 & 0.9983 \\
& 2 & 4 & 9 & 0.9617 & 16 & 17 & 0.9515 & 8 & 9 & 0.9537 & - & - & - \\
& 4 & 1 & 5 & 0.9971 & 1 & 3 & 0.9850 & 1 & 3 & 0.9932 & 1 & 2 & 0.9510 \\
0.05 & 6 & 0 & 4 & 0.9884 & 0 & 2 & 0.9787 & 0 & 2 & 0.9884 & 0 & 1 & 0.9787 \\
& 8 & 0 & 4 & 0.9973 & 0 & 2 & 0.9946 & 0 & 2 & 0.9973 & 0 & 1 & 0.9946 \\
& 10 & 0 & 4 & 0.9992 & 0 & 2 & 0.9983 & 0 & 2 & 0.9992 & 0 & 1 & 0.9983 \\
0.01 & 2 & 7 & 15 & 0.9711 & 16 & 17 & 0.9515 & 8 & 9 & 0.9537 & - & - & -
\end{tabular}




\begin{tabular}{ccccccccccccc}
4 & 1 & 7 & 0.9865 & 1 & 4 & 0.9510 & 1 & 4 & 0.9762 & 1 & 2 & 0.9510 \\
6 & 0 & 5 & 0.9793 & 1 & 4 & 0.9976 & 0 & 3 & 0.9673 & 1 & 2 & 0.9976 \\
8 & 0 & 5 & 0.9950 & 0 & 3 & 0.9840 & 0 & 3 & 0.9918 & 0 & 2 & 0.9669 \\
10 & 0 & 5 & 0.9985 & 0 & 3 & 0.9946 & 0 & 3 & 0.9974 & 0 & 2 & 0.9884 \\
\hline
\end{tabular}

Table 6: GASP for resubmitted lots having $m=3$ under SBLD for $\lambda=2.306$.

\begin{tabular}{cccccccc}
\hline \multicolumn{3}{c}{$\beta$} & \multicolumn{4}{c}{$\mathrm{r}=5$} & \multicolumn{3}{c}{$\mathrm{r}=10$} \\
& $\mu / \mu_{0}$ & \multicolumn{3}{c}{$\mathrm{a}=0.3$} & \multicolumn{3}{c}{$\mathrm{a}=0.3$} \\
& & $c$ & $g$ & $\mathrm{P}_{\mathrm{a}}\left(P_{1}\right)$ & $c$ & $g$ & $\mathrm{P}_{\mathrm{a}}\left(P_{1}\right)$ \\
\hline \multirow{4}{*}{0.25} & 2 & - & - & - & - & - & - \\
& 4 & 3 & 4 & 0.9546 & - & - & - \\
& 6 & 0 & 1 & 0.9519 & 9 & 10 & 0.9520 \\
& 8 & 0 & 1 & 0.9844 & 1 & 2 & 0.9706 \\
& 10 & 0 & 1 & 0.9941 & 0 & 1 & 0.9645 \\
& 2 & - & - & - & - & - & - \\
& 4 & 3 & 4 & 0.9546 & - & - & - \\
0.10 & 6 & 0 & 1 & 0.9519 & 9 & 10 & 0.9520 \\
& 8 & 0 & 1 & 0.9844 & 1 & 2 & 0.9706 \\
& 10 & 0 & 1 & 0.9941 & 0 & 1 & 0.9645 \\
& 2 & - & - & - & - & - & - \\
& 4 & 3 & 4 & 0.9546 & - & - & - \\
0.05 & 6 & 1 & 2 & 0.9900 & 9 & 10 & 0.9520 \\
& 8 & 1 & 2 & 0.9989 & 1 & 2 & 0.9706 \\
& 10 & 0 & 2 & 0.9645 & 0 & 1 & 0.9645 \\
& 2 & - & - & - & - & - & - \\
& 4 & 3 & 4 & 0.9546 & - & - & - \\
0.01 & 6 & 2 & 3 & 0.9976 & 9 & 10 & 0.9520 \\
& 8 & 1 & 3 & 0.9916 & 1 & 2 & 0.9706 \\
& 10 & 0 & 2 & 0.9645 & 0 & 1 & 0.9645 \\
\hline & & & & & & &
\end{tabular}

\section{Appendix B}

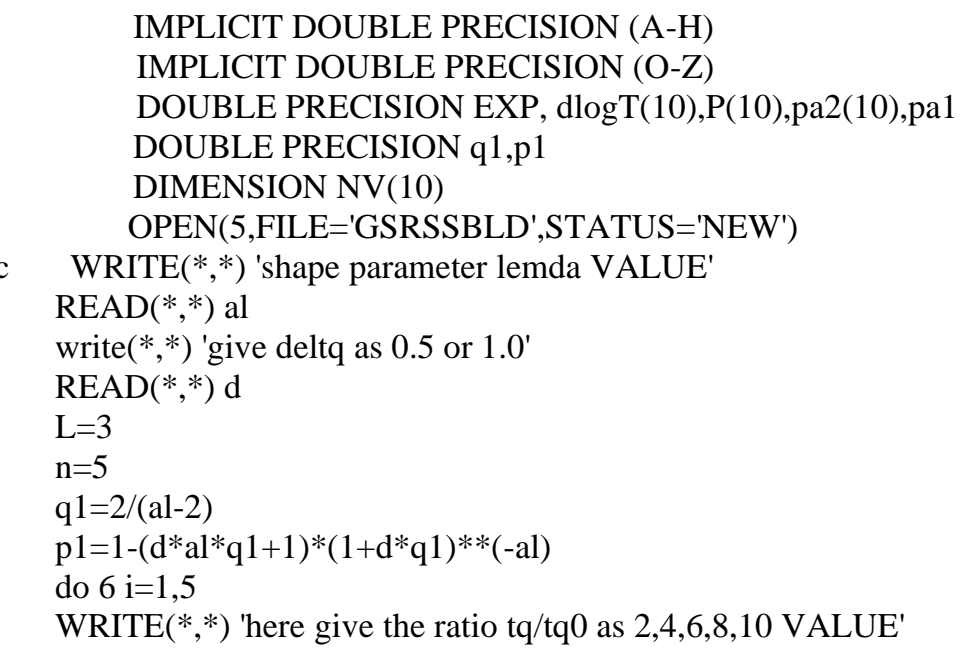




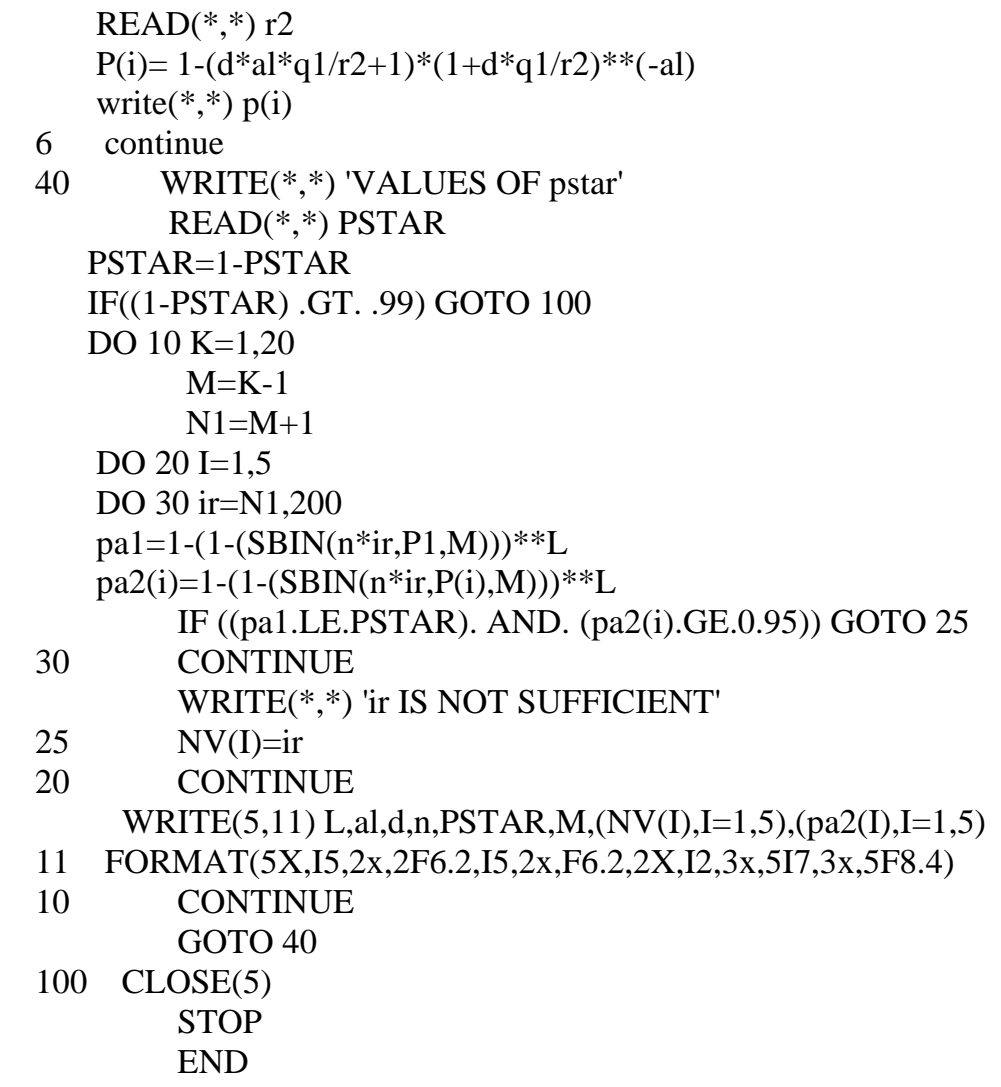

